\title{
Study on the Correlation Between ANCA and Thyroid-related Ophthalmopathy
}

\author{
Yuping Xiong ${ }^{1}$, Changfu Peng ${ }^{2, *}$ \\ ${ }^{1}$ Department of Ophthalmology, The First Affiliated Hospital of Hunan Normal University/Hunan Provincial People's Hospital, Changsha, \\ China \\ ${ }^{2}$ Department of Ophthalmology, Hunan Provincial People's Hospital/The First Affiliated Hospital of Hunan Normal University, Changsha, \\ China
}

Email address:

940971540@qq.com (Yuping Xiong),oculistpeng@yahoo.com (Changfu Peng)

*Corresponding author

\section{To cite this article:}

Yuping Xiong, Changfu Peng. Study on the Correlation Between ANCA and Thyroid-related Ophthalmopathy. International Journal of Ophthalmology \& Visual Science. Vol. 5, No. 1, 2020, pp. 21-23. doi: 10.11648/j.ijovs.20200501.14

Received: December 16, 2019; Accepted: January 2, 2020; Published: January 17, 2020

\begin{abstract}
Background The pathogenesis of thyroid associated ophthalmopathy (TAO) is still unclear. Studies have proved that there are several autoantibodies in TAO patients, and ANCA, as one of the autoantibodies, is positive in some TAO patients, but its correlation with the occurrence, development and outcome of TAO is still unclear. Objective To study the correlation between ANCA and Thyroid Associated Ophthalmopathy. Methods Prospective observational cohort study was designed. This study was approved by the Ethic Commission of Hunan Provincial People's Hospital/the First Affiliated Hospital of Hunan Normal University, and written informed consent was obtained from each Subject before entering this trial. 120 patients diagnosed as TAO and visited our hospital from January 2016 to June 2018 were enrolled in the study, including 30 cases in inactive group and 90 cases in active group [According to the criteria of clinical activity score (CAS)], 42 cases in Mild group and 78 cases in Moderate-to-severe and Sight-threatening group (According to the criteria of EUGOGO). The differences in ANCA positive rate among above groups and Active group after transferred to Inactive were compared. Results The overall positive rate of ANCA in TAO patients was $16.7 \%$. The positive rates in the Inactive group and Active group were respectively $3.33 \%$ and $21.11 \%$, and the difference was statistically significant $(P<0.05)$. The positive rates of Mild group, Moderate-to-severe group and Sight-threatening group were respectively $8.82 \%$ and $19.76 \%$, and there was no significant differences statistically $(P>0.05)$. The positive rates of Active group reduced to $5.5 \%$, and the difference was statistically significant $(P<0.05)$. Conclusion ANCA can reflects the activity of TAO to some extent, which is helpful to guide clinical treatment and prognosis judgment.
\end{abstract}

Keywords: Antineutrophil Cytoplasmic Antibody, Thyroid Associated Ophthalmopathy, Correlation

\section{Introduction}

Thyroid-related ophthalmopathy (TAO), also known as Graves ophthalmopathy (GO), is a common orbital autoimmune disease associated with Graves disease (GD) [1]. TAO is characterized by the inflammatory response of the post-bulbar tissue resulting in the excessive deposition of highly hydrophilic glycosaminoglycan (GAG) and / or the increase of post-bulb adipose tissue, which in turn leads to an increase in orbital connective tissue and extraocular muscle volume. Early pathological changes are inflammatory reactions caused by inflammatory cell infiltration and edema, and late stages are degeneration and fibrosis of tissues. The pathogenesis of TAO is currently unknown, and may be related to immunity, genetics and the environment. During the onset of TAO, inflammatory factors of orbital fibroblasts are highly secreted, such as TNF- $\alpha$, IL-6, IL-10, etc. The secretion of these inflammatory factors participates and affects the progress of TAO [2].

Studies have shown that there are several autoantigens and their corresponding serum antibodies in TAO patients, including thyroid stimulating hormone receptor (TSHR), myosin antigen, G2S, flavin protein, $\alpha$-lining protein, grape Membrane autoantigen (uveal autoantigen with coiled coil 
domains and ankyrin repeats (UACA)) and troponin (calsequesyrin) and other related antigens and corresponding antibodies [3, 4]. As a type of autoantibodies, antineutrophil cytoplasmic antibody (ANCA) is positive in some TAO patients. In this study, 120 patients with TAO in different stages and grades were taken as the research object. The correlation between ANCA and TAO was analyzed by detecting serum ANCA.

\section{Materials and Methods}

\subsection{General Information}

A total of 120 patients who were diagnosed with TAO in our hospital from June 2016 to January 2018 were collected, including 31 males and 89 females, aged 11-72 years (mean $45.25 \pm 4.63$ years). The inclusion criteria used the Bartly diagnostic criteria [5]. According to the clinical activity score (CAS) of TAO [6] (clinical activity score, CAS), patients were divided into: group A active period (90 cases, CAS score $\geq 3$ points), group B stationary period ( 30 cases, CAS score $<3$ points)); Refer to the European Group on Graves' Orbitopathy (EUGOGO) guidelines for determining the severity of disease [7] divided into mild group (42 cases), moderate to severe and threatening vision group (78 cases); included Criteria: Meet one of the following (1) + (2), (3), (4), (5); or (2) + (3), (4), (5): (1) eyelid retreat; (2) abnormal thyroid function; (3) exophthalmos; (4) extraocular muscle involvement; Exclusion criteria: (1) Combined with other eye diseases; (2) Patients with vasculitis-related diseases; (3) Those who received antithyroid treatment within half a year before the test; (4) Those who were lost to follow-up.

The study was approved by the Hunan Provincial People's Hospital / The First Affiliated Hospital of Hunan Normal University Biomedical Ethics Committee (Lot No.: 2016 Scientific Research Lun Shen (29)). All patients and their families were informed of the purpose and significance of the study and signed informed consent before the trial.

\subsection{Method}

$3 \mathrm{ml}$ venous blood was collected from each subject on an empty stomach and stored at $2-8^{\circ} \mathrm{C}$. For patients with active TAO, follow-up observations in the outpatient clinic, based on the CAS score, determine that the blood will be drawn again and tested for ANCA after entering the quiescent period. The detection method can be used to detect anti-myeloperoxidase (MPO), protease 3 (PR3), and renal small Sphere basement membrane (GMB) IgG antibody ELISA kit and indirect immunofluorescence detection ANCA kit, manufacturer: Oumeng Medical Laboratory Diagnosis Co., Ltd., the detection instrument is: EUROIMMUN Analyzer 1.

\subsection{Statistical Method}

SPSS 22.0 was used to analyze the statistics. Comparisons between groups were performed using the $\chi^{2}$ test (among them, the stationary phase and the active phase, the mild group and the moderate-to-severe, and threatened vision groups were compared using a corrected $\chi^{2}$ test), $\mathrm{P}<0.05$ was considered statistically significant.

\section{Results}

The study subjects were divided into the static period and active period group according to the CAS scoring method, and the ANCA test results (Table 1) passed the corrected $\chi^{2}$ test, and the difference between the two groups was significant $\left(\chi^{2}\right.$ $=3.92, \mathrm{P}<0.05)$.

The study subjects were divided into mild group, moderate to severe group, and threatened vision group according to the EUGOTAO grouping. The ANCA test results (Table 2) also passed the corrected $\chi^{2}$ test. And there was no significant difference between the two groups $\left(\chi^{2}=3.23, \mathrm{P}>0.05\right)$.

Comparing the ANCA test results before and after resting in active TAO patients (Table 3$)$, the difference is significant $\left(\chi^{2}\right.$ $=8.12, \mathrm{P}<0.05)$.

Table 1. ANCA test results grouped by activity.

\begin{tabular}{llll}
\hline \multirow{2}{*}{ Group } & \multicolumn{2}{c}{ ANCA results } & \multirow{2}{*}{ Total } \\
\cline { 2 - 3 } & Positive & Negative & \\
\hline Quiescent group & 1 & 29 & 30 \\
Active group & 19 & 71 & 90 \\
Total & 20 & 100 & 120 \\
\hline
\end{tabular}

Table 2. ANCA test results grouped by EUGOGO.

\begin{tabular}{llll}
\hline \multirow{2}{*}{ Group } & \multicolumn{2}{l}{ ANCA results } & \multirow{2}{*}{ Total } \\
\cline { 2 - 3 } & Positive & Negative & \\
\hline Mild & 3 & 39 & 42 \\
Moderate, severe and threatening vision & 17 & 61 & 78 \\
Total & 20 & 100 & 120 \\
\hline
\end{tabular}

Table 3. ANCA test results before and after stabilization in patients in the active group.

\begin{tabular}{llll}
\hline \multirow{2}{*}{ Group } & \multicolumn{2}{l}{ ANCA results } & \multirow{2}{*}{ Total } \\
\cline { 2 - 3 } & Positive & Negative & \\
\hline Active group (before stabilization) & 19 & 71 & 90 \\
Active group (after stabilization) & 5 & 85 & 90 \\
Total & 24 & 156 & 180 \\
\hline
\end{tabular}

\section{Discussion}

TAO is an autoimmune disease, but the specific pathogenesis is not clear. Studies have shown that there are several autoantigens and their corresponding serum antibodies in TAO patients, including TSHR, ocular myosin antigen, G2S, flavin, $\alpha$-lining protein, UACA, and troponin and other related antibodies and corresponding antibodies.

ANCA is an autoantibody that targets neutrophil granule components and monocyte lysosomes. It is divided into neutrophil cytoplasmic type (C-ANCA) and perinuclear type (PANCA) according to the site of indirect immunofluorescence [8]. It also can be used as a serological marker antibody for systemic necrotizing small vasculitis, and has certain predictive value for its remission and recurrence [9]. We found in the clinic that some TAO patients, especially 
active TAO patients, have positive serological ANCA tests, so it is unknown whether ANCA as one of the autoantibodies participates in the occurrence, development and outcome.

In this group of data, the positive rate of ANCA is $16.7 \%$, but antithyroid drugs can also cause ANCA positive. Since 1993, Dolman [10] and others first proposed [11, 12] that propylthiouracil (PTU) can cause ANCA-associated vasculitis, It has been reported at home and abroad that ANCA-positive vasculitis may occur in patients with hyperthyroidism after taking PTU. The thiourea antithyroid drugs methimazole (MMI) and PTU can cause ANCA positive, especially PTU is common, and the positive rate of ANCA caused by PTU reported in the literature can reach more than $40 \%$ [13]. Foreign Sera N [14] and other reports even reached $64 \%$. Therefore, this study excluded patients who had received antithyroid therapy within six months before the test.

In this group of data, the positive rates of the quiescent group in the mobility group were $3.33 \%$ and $21.11 \%$, respectively; the positive rates of the mild group and moderate, severe, and threatening vision groups were $8.82 \%$ and $19.76 \%$, respectively. The difference in results may be related to the lack of time for patients to stop taking antithyroid drugs after active use. After cessation of thiourea antithyroid drugs, serum ANCA levels in patients with simple ANCA-positive patients can gradually decrease to become negative, but for patients with ANCA-associated microangiitis, the ANCA-positive duration can reach several years [15]. Therefore, in the next study, it is necessary to expand the sample content and further extend the follow-up observation time, especially to observe the correlation between the time of ANCA negative and clinical outcome in TAO patients, and provide theoretical basis for clinical diagnosis and prognosis evaluation.

\section{Conclusion}

ANCA can reflects the activity of TAO to some extent, which is helpful to guide clinical treatment and prognosis judgment.

\section{Fund Project}

Project Fund of the Education Department of Hunan Province (NO: 15C0830).

\section{References}

[1] Xiong Wei, Tan Jia, Peng Changfu. Individualized comprehensive diagnosis and treatment of thyroid-related ophthalmopathy [M]. Changsha: Central South University Press, 2014: 1-19.

[2] Qian Yu, Li Yuzhen, Liang Wenjun, et al. Research and treatment progress of thyroid-related ophthalmopathy $[\mathrm{J}]$.
Advances in Modern Biomedicine 2017; 17 (16): 3195-3200. DOI: 10.13241/j.cnki.pmb.2017.16.048.

[3] Fu Dian, Liu Yi, Wang Jian. Research progress on thyroid-related ophthalmopathy and autoantigens [J]. Medical Review, 2012, $18 \quad$ (9): 1374-1376. DOI: 10.3969/j.issn.1006-2084.2012.09.035.

[4] Feng Xiaoting, Li Zhangfang, Shen Jie: Progress in the diagnosis and treatment of thyroid-related ophthalmopathy. [J]. Chinese Journal of Practical Internal Medicine 2019, 39 (04): 384-388.

[5] Luoqing Li TAO [M] Beijing: People's Medical Publishing House, 2005: 301.

[6] Stan MN, Garrity JA, Bahn RS. The evaluation and treatment of graves ophthalmopathy [J]. Med Clin North Am, 2012, 96 (2): 311-28. DOI: 10.1016/j.mcna.2012.01.014.

[7] Bartalena L, Baldeschi L, Boboridis K. The 2016 European Thyroid Association/European Group on Graves' Orbitopathy Guidelines for the Management of Graves Orbitopathy [J]. Eur Thyroid J, 2016, 5 (1): 9-26. DOI: 10.1159/000443828.

[8] Tan Liming, Jiao Anjun, Feng Xiaojing, Xu Yanyue, Tan Fuyan, He Siqi, Luo Yan, Chen Juanjuan, Jiang Yongqing, Li Hua: Clinical value of antineutrophil cytoplasmic antibody detection for systemic vasculitis. [J]. Laboratory Medicine 2018, 33 (02): 101-105.

[9] Li Li, Ruofen Wu. Detection and significance of anti-neutrophil cytoplasmic antibodies [J]. Ningxia Medical Journal, 2006, 28 (6): 435-436. DOI: 1001-5949(2006)06-0435-02.

[10] Dolman KM, Gans RO, Vervaat TJ, et al. Vasculitis and antineutrophil cytoplasmic autoantibodies associated with propylthiouracil therapy [J]. Lancet, 1993:, 342: 651-652. DOI: 10.1016/0140-6736(93)91761-A.

[11] Wei Yun, Tang Wei: 1 case of anti-neutrophil cytoplasmic antibody-associated vasculitis caused by methimazole. [J]. Zhongnan Pharmaceutical 2019, 17 (11): 2007-2009.

[12] Shi Zhuo, Fan Zhongmin, Xia Zhengkun, Gao Yuanfu, Sun Tao, Huang Qian, Ren Xianguo, Zhang Pei: Children with secondary anti-neutrophil cytoplasmic antibody-associated microangiitis caused by propylthiouracil: a case report and literature review. [J]. Clinical Pediatrics 2012, 30 (04): 316-318.

[13] Gao Hongwei. Evaluation of the efficacy and safety of antithyroid drugs in thyroid diseases [J]. Drug and Clinical Medicine, 2015, 12 (9): 35-39.

[14] Sera N, Ashizawa K, Ando T, et al. Treatment with propylthiouracil is associated with appearance of antineutrophil cytoplasmic antibodies in some patients with Graves' disease [J]. Thyroid, 2000, 10: 595-599. DOI: $10.1089 /$ thy.2000.10.595.

[15] Liu Chao, Jiang Lin. Recognition of adverse reactions to antithyroid drugs [J]. Chinese Journal of Endocrinology and Metabolism, 2011, 27 (6): 529-532. DOI: 10.3760/cma.j.issn.1000-6699.2011.06.022. 